\title{
On Two Covariates Cosine and Sine Noisy-Wave Trigonometry Regression of Heartbeats
}

\author{
Olanrewaju Rasaki Olawale \\ Department of Statistics, Faculty of Science, University of Ibadan, Ibadan, Oyo State, Nigeria
}

\begin{abstract}
This paper proposes and describes the acumen on alternate two covariates linear Cosine and Sine regression functions that possessed a noisy-wave or tone frequencies via wave-trend of actualized observations of regressors and responsive variable needed in fitting a wavy equation of trigonometry regression. The method of maximum likelihood was used in estimating parameters associated to the Cosine and Sine alternate functions via vector coefficients as well as their distributional and residual properties. The estimations obtained via the method were enthralled to the noisy-wave mesokurtic observations of babies' rate of heartbeats exactly an hour after birth $\left(\mathrm{HR}_{1}\right)$, two hours after birth $\left(\mathrm{HR}_{2}\right)$ and three hours after birth $\left(\mathrm{HR}_{3}\right)$. The implementation and illustrative application was via $\mathrm{R}$ using the heartbeat dataset. It was gleaned that the trigonometry equation line of $\alpha+\beta_{1} \cos \left(\mathrm{HR}_{2}\right)+\beta_{2} \sin \left(\mathrm{HR}_{1}\right)$ optimally captured the wave observations and robustly outstripped the alternate Cosine and Sine equation line of $\alpha+\beta_{1} \sin \left(\mathrm{HR}_{2}\right)+\beta_{2} \cos \left(\mathrm{HR}_{1}\right)$.
\end{abstract}

Keywords: Cosine; Equation line; Noisy-wave; Regression functions; Rates of heartbeat; Sine.

(9) (1) CC BY: Creative Commons Attribution License 4.0

\section{Introduction}

Regression analysis is a technique use in modeling the relationship(s) between response variable and predictor(s) or among predictors. This unknown connection could either be a linear or non-linear relationship depending on the transfer function $[1,2]$. It is termed "simple regression" if the dependent variable is constrained to only a predictor and "multiple regression" if the formal is subjugated to two or more predictors [3]. The conventional methods of statistic and parameter (regression coefficients, model performance indexes, residual indexes, prediction error indexes, etc.) estimation ranges from Maximum Likelihood (ML), Least Squares (LS), Quasi- Likelihood (QL), Generalized Linear Model (GLM) etc. for parametric approach; method of sieves, difference sequence method, Ordinary differential Equations (ODEs) etc. for non-parametric approach and some amalgamated methods of both parametric and non-parametric that resulted in semi-parametric approach [4-7]. The main purpose of regression modeling is for generalization of studied relationship(s), prediction making, decision-making, diagnosis and to ascertain statistical property of the studied system $[8,9]$.

According to Hanley [10], a number of extensive studies had been carried-out on different forms of regression estimators to accommodate and recodify the assumptions of normality, independence and attached time factors to covariates. Among the few forms are ridge regression, seasonality regression analysis, Fourier regression, trigonometric series regression analysis, and smoothing splines regression [11-14]. All these mentioned forms are for demonstrating the dummy variables for estimation of seasonal effects in a time series, to penalize estimators in situation where the number of parameters estimated is strictly greater than the sample size, and to free the distributional property of the observations in non-parametric settings [15-17].

Rigdon, et al. [18], propounded a Fourier trigonometric like regression and applied it to uniform time-varying public health surveillance disease data with the assertion of normality assumption, seasonality, and independence ascertained as well as the stationarity of the first and second order- Fourier regression like model.

This paper presents a conspectus diversify approach by considering noisy-wave or tone frequencies observations of covariates without seasonality, uniform time varying (unequal spaced time intervals of unordered sequence of set of observations) of recording observations via a Gaussian density function. A two alternate Cosine and Sine linear equation functions (a trigonometry regression approach) will be formulated such the parametric method of maximum likelihood will adopted in estimating the Cosine and Sine alternate equations vector coefficient noisy-wave mesokurtic observations as well as its distributional and residual traits.

\section{Material and Method}

\subsection{The Two Covariates Alternate Cosine and Sine Function Trigonometry Regression}

Given a linear regression model function with random error variables $\omega_{i}$;

$$
Y=f(X \beta)+\omega_{i}
$$

For ${ }^{\omega_{i}}$ are uncorrelated noisy-wave standardized random variables with mean zero and unity variance. 
And,

$$
f(X \beta)=\alpha+\beta_{1} \cos X_{i 1}+\beta_{2} \sin X_{i 2}
$$

Then,

$$
y_{i}=\alpha+\beta_{1} \cos X_{i 1}+\beta_{2} \sin X_{i 2}+\omega_{i}
$$

Alternatively;

$y_{i}=\alpha+\beta_{1} \sin X_{i 1}+\beta_{2} \cos X_{i 2}+\omega_{i}$

Where,

$y_{i} \Rightarrow$ Is a $n b y 1_{\text {vector of responses. }}$

$X_{i 1}, X_{i 2} \Rightarrow$ Is a $n$ by $p+1$ is full rank design matrix of the model.

$\beta=\alpha, \beta_{1}, \beta_{2} \Rightarrow$ Is a $p b y 1$ vector of coefficients.

$\omega_{i} \Rightarrow$ Is a $n b y 1_{\text {vector of random errors. }}$

$\operatorname{Cos} \& \operatorname{Sin} \Rightarrow$ Are the trigonometry noisy-waves for the two covariates.

$$
\omega_{i}=y-\alpha-\beta_{1} \cos X_{i 1}-\beta_{2} \sin X_{i 2}
$$

In matrix form;

$$
Y=H B+\omega
$$

$B$ Is the column vector of parameter to be estimated; $H$ is the coefficient matrix of a square matrix; where the error term $\omega \square\left(0, I \sigma_{\omega}^{2}\right)$

\subsection{Maximum Likelihood Estimation Method of the Two Covariates Alternate Cosine and Sine Function}

Considering error random variables $\left(\omega_{i}\right)$ that are assumed independent and normally distributed with zero mean and unity variance, adopting the maximum likelihood estimation gives

$$
f(\omega)=\frac{1}{\sqrt{2 \pi \sigma^{2}}} \exp \left[\frac{1}{2 \sigma^{2}}(\omega)^{2}\right]
$$

Then,

$$
f(\omega)=\frac{1}{\sqrt{2 \pi \sigma^{2}}} \exp \left[\frac{1}{2 \sigma^{2}}\left(y_{i}-\alpha-\beta_{1} \cos X_{i 1}-\beta_{2} \sin X_{i 2}\right)^{2}\right]
$$

The maximum likelihood gives,

$$
\begin{gathered}
L=f\left(\omega_{1}\right) \times f\left(\omega_{2}\right) \times \cdots \times \times f\left(\omega_{n}\right) \\
L=\left(\frac{1}{\sqrt{2 \pi \sigma^{2}}}\right)^{n} \exp \sum_{i=1}^{n}\left[\frac{1}{2 \sigma^{2}}\left(y_{i}-\alpha-\beta_{1} \cos X_{i 1}-\beta_{2} \sin X_{i 2}\right)^{2}\right] \\
\ln L=-n \ln \sigma-\frac{1}{2} \ln (2 \pi)-\frac{1}{2} \sum_{i=1}^{n}\left[\frac{\left(y_{i}-\alpha-\beta_{1} \cos X_{i 1}-\beta_{2} \sin X_{i 2}\right)}{\sigma}\right]^{2} \\
\text { Taking the } \log \text { of equation }
\end{gathered}
$$

$$
\frac{\partial \ln L}{\partial \alpha}=\sum_{i=1}^{n}\left[\frac{\left(y_{i}-\alpha-\beta_{1} \cos X_{i 1}-\beta_{2} \sin X_{i 2}\right)}{\sigma}\right]
$$

Equating to zero gives,

$$
\sum_{i=1}^{n}\left(y_{i}-\alpha-\beta_{1} \cos X_{i 1}-\beta_{2} \sin X_{i 2}\right)=0
$$

Equating to zero gives,

$$
\frac{\partial \ln L}{\partial \beta_{1}}=\cos X_{i 1} \sum_{i=1}^{n}\left[\frac{\left(y_{i}-\alpha-\beta_{1} \cos X_{i 1}-\beta_{2} \sin X_{i 2}\right)}{\sigma}\right]
$$


Equating to zero gives,

$$
\begin{gathered}
\sum_{i=1}^{n} \cos X_{i 1}\left(y_{i}-\alpha-\beta_{1} \cos X_{i 1}-\beta_{2} \sin X_{i 2}\right)=0 \\
\frac{\partial \ln L}{\partial \beta_{2}}=\sin X_{i 2} \sum_{i=1}^{n}\left[\frac{\left(y_{i}-\alpha-\beta_{1} \cos X_{i 1}-\beta_{2} \sin X_{i 2}\right)}{\sigma}\right]
\end{gathered}
$$

$$
\sum_{i=1}^{n} \sin X_{i 2}\left(y_{i}-\alpha-\beta_{1} \cos X_{i 1}-\beta_{2} \sin X_{i 2}\right)=0
$$

Expanding equations (7), (8) and (9) gives the system of equations;

$$
\begin{gathered}
\sum_{i=1}^{n} y_{i}-n \alpha-\beta_{1} \sum_{i=1}^{n} \cos X_{i 1}-\beta_{2} \sum_{i=1}^{n} \sin X_{i 2}=0 \\
\sum_{i=1}^{n}\left(y_{i} \cos X_{i 1}\right)-\alpha \sum_{i=1}^{n}\left(\cos X_{i 1}\right)-\beta_{1} \sum_{i=1}^{n}\left(\cos ^{2} X_{i 1}\right)-\beta_{2} \sum_{i=1}^{n}\left(\cos X_{i 1}\right)\left(\sin X_{i 2}\right)=0 \\
\sum_{i=1}^{n}\left(y_{i} \sin X_{i 2}\right)-\alpha \sum_{i=1}^{n}\left(\sin X_{i 2}\right)-\beta_{1} \sum_{i=1}^{n}\left(\sin X_{i 2}\right)\left(\cos X_{i 1}\right)-\beta_{2} \sum_{i=1}^{n}\left(\sin ^{2} X_{i 2}\right)=0
\end{gathered}
$$

Re-arranging and converting to matrix form gives,

$$
\left(\begin{array}{c}
\sum_{i=1}^{n} y_{i} \\
\sum_{i=1}^{n}\left(y_{i} \cos X_{i 1}\right) \\
\sum_{i=1}^{n}\left(y_{i} \sin X_{i 2}\right)
\end{array}\right)=\left(\begin{array}{c}
\alpha \\
\beta_{1} \\
\beta_{2}
\end{array}\right)\left(\begin{array}{ccc}
n & \sum_{i=1}^{n} \cos X_{i 1} & \sum_{i=1}^{n} \sin X_{i 2} \\
\sum_{i=1}^{n}\left(\cos X_{i 1}\right) & \sum_{i=1}^{n}\left(\cos ^{2} X_{i 1}\right) & \sum_{i=1}^{n}\left(\cos X_{i 1}\right)\left(\sin X_{i 2}\right) \\
\sum_{i=1}^{n}\left(\sin X_{i 2}\right) & \sum_{i=1}^{n}\left(\sin X_{i 2}\right)\left(\cos X_{i 1}\right) & \sum_{i=1}^{n}\left(\sin ^{2} X_{i 2}\right)
\end{array}\right)
$$

Where,

$$
\begin{aligned}
& B=\left(\begin{array}{l}
\alpha \\
\beta_{1} \\
\beta_{2}
\end{array}\right)=\left(\begin{array}{ccc}
n & \sum_{i=1}^{n} \cos X_{i 1} & \sum_{i=1}^{n} \sin X_{i 2} \\
\sum_{i=1}^{n}\left(\cos X_{i 1}\right) & \sum_{i=1}^{n}\left(\cos ^{2} X_{i 1}\right) & \sum_{i=1}^{n}\left(\cos X_{i 1}\right)\left(\sin X_{i 2}\right) \\
\sum_{i=1}^{n}\left(\sin X_{i 2}\right) & \sum_{i=1}^{n}\left(\sin X_{i 2}\right)\left(\cos X_{i 1}\right) & \sum_{i=1}^{n}\left(\sin ^{2} X_{i 2}\right)
\end{array}\right)^{-1}\left(\begin{array}{c}
\sum_{i=1}^{n} y_{i} \\
\sum_{i=1}^{n}\left(y_{i} \cos X_{i 1}\right) \\
\sum_{i=1}^{n}\left(y_{i} \sin X_{i 2}\right)
\end{array}\right) \\
& B=\left(H^{T} H\right)^{-1} H^{T} y
\end{aligned}
$$

Where,

$$
\left(H^{T} H\right)^{-1}=\left(\begin{array}{ccc}
n & \sum_{i=1}^{n} \cos X_{i 1} & \sum_{i=1}^{n} \sin X_{i 2} \\
\sum_{i=1}^{n}\left(\cos X_{i 1}\right) & \sum_{i=1}^{n}\left(\cos ^{2} X_{i 1}\right) & \sum_{i=1}^{n}\left(\cos X_{i 1}\right)\left(\sin X_{i 2}\right) \\
\sum_{i=1}^{n}\left(\sin X_{i 2}\right) & \sum_{i=1}^{n}\left(\sin X_{i 2}\right)\left(\cos X_{i 1}\right) & \sum_{i=1}^{n}\left(\sin ^{2} X_{i 2}\right)
\end{array}\right)^{-1}
$$$$
H^{T} y=\left(\begin{array}{c}
\sum_{i=1}^{n} y_{i} \\
\sum_{i=1}^{n}\left(y_{i} \cos X_{i 1}\right) \\
\sum_{i=1}^{n}\left(y_{i} \sin X_{i 2}\right)
\end{array}\right)
$$

Since cosine of $x_{i}$ varies from sine of $x_{i}$, that is $\cos \left(x_{i}\right) \neq \sin \left(x_{i}\right)$ 


$$
\begin{gathered}
y_{i}=\alpha+\beta_{1} \sin X_{i 1}+\beta_{2} \cos X_{i 2}+\omega_{i} \\
\omega_{i}=y_{i}-\alpha-\beta_{1} \sin X_{i 1}-\beta_{2} \cos X_{i 2}
\end{gathered}
$$

When $y_{i}=\alpha+\beta_{1} \sin X_{i 1}+\beta_{2} \cos X_{i 2}+\omega_{i}$, the estimates of equation (13) becomes equation (17) below;

$$
B=\left(\begin{array}{l}
\alpha \\
\beta_{1} \\
\beta_{2}
\end{array}\right)=\left(\begin{array}{ccc}
n & \sum_{i=1}^{n} \sin X_{i 1} & \sum_{i=1}^{n} \cos X_{i 2} \\
\sum_{i=1}^{n}\left(\sin X_{i 1}\right) & \sum_{i=1}^{n}\left(\sin ^{2} X_{i 1}\right) & \sum_{i=1}^{n}\left(\sin X_{i 1}\right)\left(\cos X_{i 2}\right) \\
\sum_{i=1}^{n}\left(\cos X_{i 2}\right) & \sum_{i=1}^{n}\left(\cos X_{i 2}\right)\left(\sin X_{i 1}\right) & \sum_{i=1}^{n}\left(\cos ^{2} X_{i 2}\right)
\end{array}\right)^{-1}\left(\begin{array}{c}
\sum_{i=1}^{n} y_{i} \\
\sum_{i=1}^{n}\left(y_{i} \sin X_{i 1}\right) \\
\sum_{i=1}^{n}\left(y_{i} \cos X_{i 2}\right)
\end{array}\right)
$$

\subsection{Distributional Properties of the Two Covariates Trigonometric Regression}

From equation (14)

$$
B=\left(H^{T} H\right)^{-1} H^{T} y
$$

Recall from equation (4), $\quad y=H B+\omega$

$$
\begin{array}{rr}
\text { So, } & B=\left(H^{T} H\right)^{-1} H^{T}(H B+\omega) \\
=B+H^{T} \omega & =\left(H^{T} H\right)^{-1}\left(H^{T} H\right) B+H^{T} \omega
\end{array}
$$

Taking expectation gives,

$$
\begin{aligned}
& E(B)=E(B)+H^{T} E(\omega) H \\
& \text { Recall } E(\omega)=0,
\end{aligned}
$$$$
E(B)=B
$$

So, mean of the estimate $B$ is nothing but $B$

Subtracting " $B$ " from both sides of equation (14) gives

$$
\begin{gathered}
B-B=H^{T} \omega \quad \text { But, } \quad y-H B=\omega \\
B-B=H^{T}(y-H B) \\
B-B=H^{T} y-H^{T} H B=\left(H^{T} H\right)^{-1} H \omega
\end{gathered}
$$

\subsection{The Dispersion Matrix of $B$}

$$
\begin{gathered}
V(B)=E[B-B]^{T}[B-B] \\
=E\left[\left(H^{T} H\right)^{-1} H \omega\right] \\
=E\left[\left(H^{T} H\right)^{-1} H \omega \omega^{T} H\left(H^{T} H\right)^{-1}\right] \\
=\left[\left(H^{T} H\right)^{-1} H E\left(\omega \omega^{T}\right) H\left(H^{T} H\right)^{-1}\right]
\end{gathered}
$$

Recall $\quad E\left(\omega \omega^{T}\right)=\sigma_{\omega}^{2}$

$$
=\left[\left(H^{T} H\right)^{-1} H H\left(H^{T} H\right)^{-1}\right] \sigma_{\omega}^{2}
$$

$$
V(B)=\left(H^{T} H\right)^{-1} \sigma_{\omega}^{2}
$$

Where $\sigma_{\omega}^{2}$ is the variance of the error term 
This implies that,

$$
B \square\left(B,\left(H^{T} H\right)^{-1} \sigma_{\omega}^{2}\right)
$$

Such tha

$$
V(B)=\left(\begin{array}{ccc}
n & \sum_{i=1}^{n} \cos X_{i 1} & \sum_{i=1}^{n} \sin X_{i 2} \\
\sum_{i=1}^{n}\left(\cos X_{i 1}\right) & \sum_{i=1}^{n}\left(\cos ^{2} X_{i 1}\right) & \sum_{i=1}^{n}\left(\cos X_{i 1}\right)\left(\sin X_{i 2}\right) \\
\sum_{i=1}^{n}\left(\sin X_{i 2}\right) & \sum_{i=1}^{n}\left(\sin X_{i 2}\right)\left(\cos X_{i 1}\right) & \sum_{i=1}^{n}\left(\sin ^{2} X_{i 2}\right)
\end{array}\right)^{-1} \sigma_{\omega}^{2}
$$

$$
\text { For } y_{i}=\alpha+\beta_{1} \cos X_{i 1}+\beta_{2} \sin X_{i 2}+\omega_{i}
$$

While

$$
V(B)=\left(\begin{array}{ccc}
n & \sum_{i=1}^{n} \sin X_{i 1} & \sum_{i=1}^{n} \cos X_{i 2} \\
\sum_{i=1}^{n}\left(\sin X_{i 1}\right) & \sum_{i=1}^{n}\left(\sin ^{2} X_{i 1}\right) & \sum_{i=1}^{n}\left(\sin X_{i 1}\right)\left(\cos X_{i 2}\right) \\
\sum_{i=1}^{n}\left(\cos X_{i 2}\right) & \sum_{i=1}^{n}\left(\cos X_{i 2}\right)\left(\sin X_{i 1}\right) & \sum_{i=1}^{n}\left(\cos ^{2} X_{i 2}\right)
\end{array}\right)^{-1} \sigma_{\omega}^{2}
$$

2.5. Variance of the Error Term for the Two the Two Covariates Trigonometric Regression

$$
\sigma_{\omega}^{2}=\frac{E\left(\omega^{T} \omega\right)}{n-p}=\frac{y^{T} y-B H^{T} y}{n-p}
$$

Where $" n "$ is the number of observations and $" p "$ is the number of parameter to be estimated.

$$
\sigma_{\omega}^{2}=\frac{y^{T} y-\left(\alpha, \beta_{1}, \beta_{2}\right)\left(\begin{array}{c}
\sum_{i=1}^{n} y_{i} \\
\sum_{i=1}^{n}\left(y_{i} \cos X_{i 1}\right) \\
\sum_{i=1}^{n}\left(y_{i} \sin X_{i 2}\right)
\end{array}\right)}{n-p}
$$

For $y_{i}=\alpha+\beta_{1} \cos X_{i 1}+\beta_{2} \sin X_{i 2}+\omega_{i}$

$$
\sigma_{\omega}^{2}=\frac{y^{T} y-\left(\alpha, \beta_{1}, \beta_{2}\right)\left(\begin{array}{c}
\sum_{i=1}^{n} y_{i} \\
\sum_{i=1}^{n}\left(y_{i} \sin X_{i 1}\right) \\
\sum_{i=1}^{n}\left(y_{i} \cos X_{i 2}\right)
\end{array}\right)}{n-p}
$$

For $y_{i}=\alpha+\beta_{1} \sin X_{i 1}+\beta_{2} \cos X_{i 2}+\omega_{i}$

\subsection{Coefficient of Determination for the two Covariates Trigonometric Regression}

The coefficient of determination being denoted by; 


$$
\begin{aligned}
& R^{2}=\frac{B H^{T} y-n \bar{y}^{2}}{y^{T} y-n \bar{y}} \\
& R^{2}=\frac{\left(\alpha, \beta_{1}, \beta_{2}\right)\left(\begin{array}{c}
\sum_{i=1}^{n} y_{i} \\
\sum_{i=1}^{n}\left(y_{i} \cos X_{i 1}\right) \\
\sum_{i=1}^{n}\left(y_{i} \sin X_{i 2}\right)
\end{array}\right)-n \bar{y}^{2}}{y^{T} y-n \bar{y}^{2}} \\
& \text { For } y_{i}=\alpha+\beta_{1} \cos X_{i 1}+\beta_{2} \sin X_{i 2}+\omega_{i} \\
& R^{2}=\frac{\left(\alpha, \beta_{1}, \beta_{2}\right)\left(\begin{array}{c}
\sum_{i=1}^{n} y_{i} \\
\sum_{i=1}^{n}\left(y_{i} \sin X_{i 1}\right) \\
\sum_{i=1}^{n}\left(y_{i} \cos X_{i 2}\right)
\end{array}\right)-n \bar{y}^{2}}{y^{T} y-n \bar{y}^{2}} \\
& \text { For } y_{i}=\alpha+\beta_{1} \sin X_{i 1}+\beta_{2} \cos X_{i 2}+\omega_{i}
\end{aligned}
$$

\section{Results}

The secondary dataset used in validating the obtained estimations above was the readings of rate of heartbeats of newly born babies in Lagos University Teaching Hospital (LUTH), a federal government owned hospital in Lagos state, Nigeria. These rate of heartbeats' readings variability were recorded in three different time-frames (in hours); rate of heartbeats exactly after an hour after birth $\left(\mathrm{HR}_{1}\right)$, rate of heartbeats exactly after two hours after birth $\left(\mathrm{HR}_{2}\right)$ and rate of heartbeats exactly after three hours after birth $\left(\mathrm{HR}_{3}\right)$. These readings were recorded for nine hundred and fifty (950) babies in the year 2017. These readings were examined and recorded via Electrocardiogram (ECG). HR $_{1}$ and $\mathrm{HR}_{2}$ are considered the two covariates (independent variables) because of the fact that the responses of $\mathrm{HR}_{3}$ rely solely upon the improved heartbeats of the first two hours after birth.

Figure-1. The Noisy-Wave of Heartbeats of $\mathrm{HR}_{1}$ and $\mathrm{HR}_{2}$

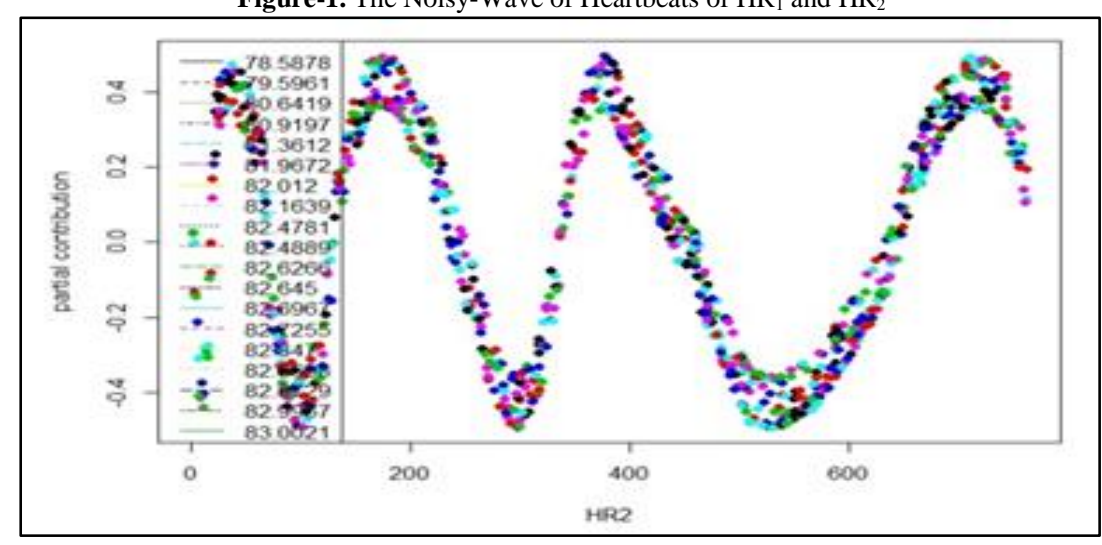

Figure-2. The Partial Cosine and Sine Wave Trend of $\mathrm{HR}_{1}$ and $\mathrm{HR}_{2}$ 

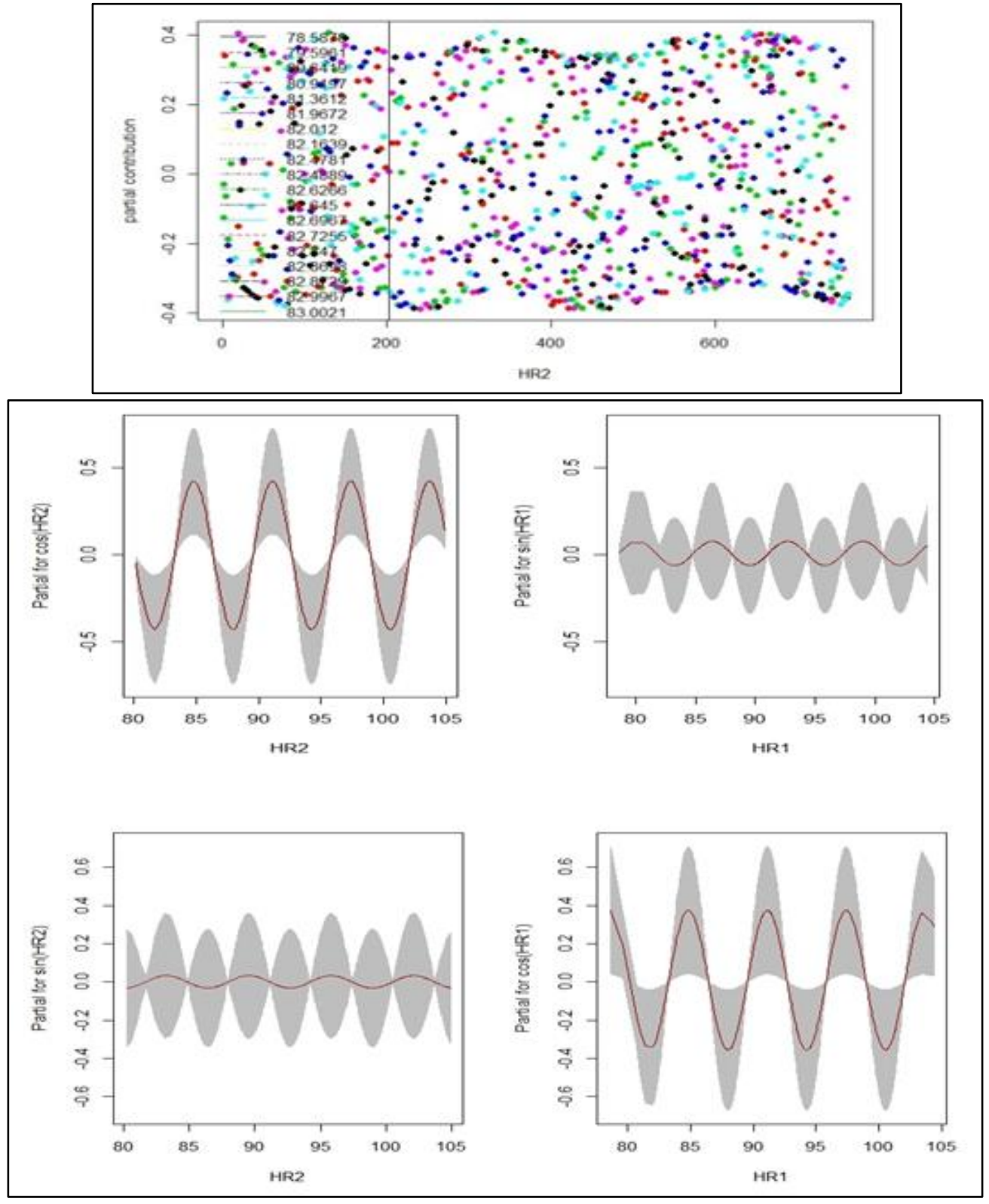

From Figure 1 and 2, the trend of the actual readings of the rate of heartbeats exactly one and two hours after birth, that is, $\mathrm{HR}_{1}$ and $\mathrm{HR}_{2}$ for the same level of four mesokurtics (Normal bell-curves) nature possessed. The Sine and Cosine plots of the two readings (the two covariates) revealed and actualized the possessed noisy-wave (Sine and Cosine waves) of the two examined observations of babies' heartbeats. This suggested a wave particle duality of the heartbeats. In other words, the $\mathrm{HR}_{1}, \mathrm{HR}_{2}$ and $\mathrm{HR}_{3}$ heartbeats are noise or tone frequencies, that is, noisy data (noisy-wave) that requested a trigonometry (Cosine and Sine) transformation or Fourier transformation as an alternative to smoothing process or modeling.

\begin{tabular}{l|l|l|l|l}
\multicolumn{5}{l}{ Table-1. Fitted Cosine and Sine equation of $\alpha+\beta_{1} \cos \left(\mathrm{HR}_{2}\right)+\beta_{2} \sin \left(\mathrm{HR}_{1}\right)$} \\
\hline Parameter & Estimate & Std. Error & t-value & $\operatorname{Pr} .(>|\mathbf{t}|)$ \\
\hline$\alpha$ & 58.6749 & 0.1112 & 527.647 & $<0.0021$ \\
\hline$\beta_{1}$ & -0.4261 & 0.1539 & -2.769 & 0.0057 \\
\hline$\beta_{2}$ & -0.0692 & 0.1525 & -0.454 & 0.6501 \\
\hline$\sigma_{\omega}$ & 1.2220 & 0.0229 & 53.27 & $<0.0021$ \\
\hline
\end{tabular}

Global Deviance: 17.802

AIC: $\quad 5025.802$

SBC: $\quad 5045.228$

log Lik: -2508.901

The Maximum Likelihood estimator is 281.132

$$
\begin{gathered}
\mathrm{HR}_{3}=58.6749-0.4261 \cos \left(\mathrm{HR}_{2}\right)-0.0692 \sin \left(\mathrm{HR}_{1}\right) \\
\text { Such that, } \sigma_{\omega} \square(1.2220,0.00052)
\end{gathered}
$$


Table-2. Fitted Cosine and Sine equation of $\alpha+\beta_{1} \sin \left(\mathrm{HR}_{2}\right)+\beta_{2} \cos \left(\mathrm{HR}_{1}\right)$

\begin{tabular}{l|l|l|l|l}
\hline Parameter & Estimate & Std. Error & t-value & Pr. $(>|\mathbf{t}|)$ \\
\hline$\alpha$ & 58.6837 & 0.1105 & 531.246 & $<0.0021$ \\
\hline$\beta_{1}$ & 0.0325 & 0.1586 & 0.205 & 0.8378 \\
\hline$\beta_{2}$ & -0.3650 & 0.1619 & -2.255 & 0.0244 \\
\hline$\sigma_{\omega}$ & 1.2235 & 0.0229 & 53.33 & $<2 \mathrm{e}-16$ \\
\hline
\end{tabular}

Global Deviance: 20.719

AIC: 5028.719

SBC: 5048.144

log Lik: -2510.359

The Maximum Likelihood estimator is 43.47365

$$
\mathrm{HR}_{3}=58.6837+0.0325 \sin \left(\mathrm{HR}_{2}\right)-0.3650 \cos \left(\mathrm{HR}_{1}\right)
$$

$$
\text { Such that } \sigma_{\omega} \square(1.2235,0.00052)
$$

Table.1 was subjected to the fitted function of $\alpha+\beta_{1} \cos \left(\mathrm{HR}_{2}\right)+\beta_{2} \sin \left(\mathrm{HR}_{1}\right)$, while table. 2 was based on $\alpha+\beta_{1} \sin \left(\mathrm{HR}_{2}\right)+\beta_{2} \cos \left(\mathrm{HR}_{1}\right)$

It was deduced that the formal fitted equation robustly accommodated the wave like nature with improved model performance of (AIC: 5025.802; SBC: 5045.228) compare to a less model performance of (AIC: 5028.719; SBC: 5048.144) by the latter. Furthermore, the global aberrances from normal nontrigonometry fitted line of the two alternate Cosine and Sine equations were relatively miniature in the two fitted equations, with a lesser miniature of global deviance of 17.802 in fitted $\alpha+\beta_{1} \cos \left(\mathrm{HR}_{2}\right)+\beta_{2} \sin \left(\mathrm{HR}_{1}\right)$ compare to a global deviance of 17.802 in fitted $\alpha+\beta_{1} \sin \left(\mathrm{HR}_{2}\right)+\beta_{2} \cos \left(\mathrm{HR}_{1}\right)$. In collaboration with the stated claims, the coefficient of $\beta_{1}$, which was the estimated coefficient of the rate of heartbeats exactly after an hour after birth $\left(\mathrm{HR}_{1}\right)$ in the formal equation hinted to be the most significant co-variate in the contributing factor to the next stability of heartbeats of babies in the next three hours and more after birth. This is due to its $\mathrm{P}$ value $=0.0057$ being strictly far away from the $5 \%$ chance of error. In the latter, it was the coefficient of $\beta_{2}$ for rate of heartbeats exactly after an hour after birth $\left(\mathrm{HR}_{2}\right)$ with $\mathrm{P}$-value $=0.0244$ that was greater than the $\mathrm{P}-\mathrm{value}=0.0057$ of the latter.

Figure-3. The Residual Deviance of the fitted Equations of $\alpha+\beta_{1} \cos \left(\mathbf{H R}_{2}\right)+\boldsymbol{\beta}_{2} \sin \left(\mathbf{H R} \mathbf{R}_{1}\right)$ and $\alpha+\beta_{1} \sin \left(\mathrm{HR}_{2}\right)+\beta_{2} \cos \left(\mathrm{HR}_{1}\right)$

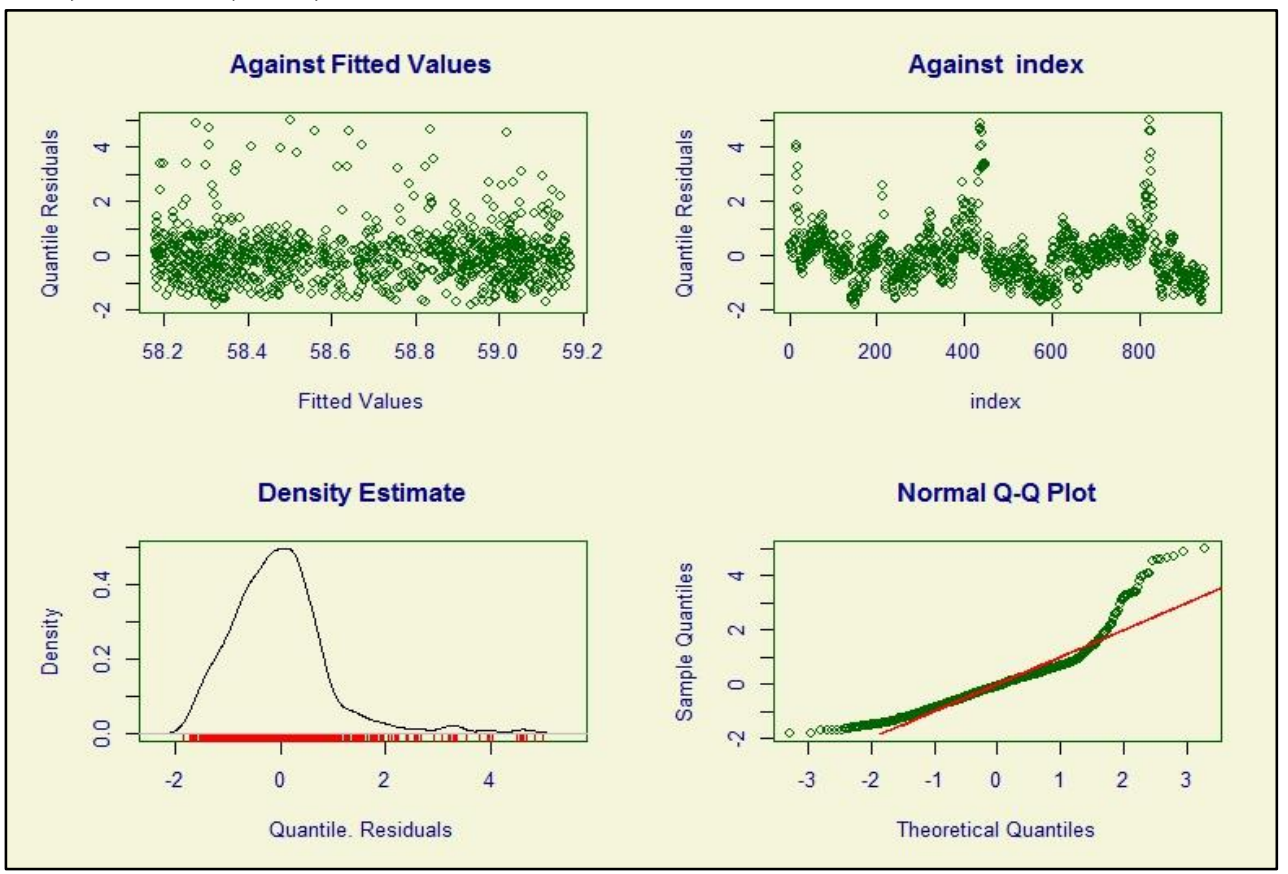

It was noted that the two alternate fitted functions of Cosine and Sine yielded the same residual indexes in terms of the estimated quantiles density, QQ-plot and approximately the same the observed and estimated frequencies. 
Table-3. Centile of the Cosine and Sine alternate equation lines

\begin{tabular}{l|l|l}
\hline Centiles & $\begin{array}{c}\alpha+\beta_{1} \cos \left(\mathrm{HR}_{2}\right)+ \\
\beta_{2} \sin \left(\mathrm{HR}_{1}\right)\end{array}$ & $\begin{array}{c}\alpha+\beta_{1} \sin \left(\mathrm{HR}_{2}\right)+ \\
\beta_{2} \cos \left(\mathrm{HR}_{1}\right)\end{array}$ \\
\hline \% of cases below 0.4 centile is & 0 & 0 \\
\hline \% of cases below 10 centile is & 6 & 6.1053 \\
\hline \% of cases below 50 centile is & 54.4211 & 54.42105 \\
\hline \% of cases below 90 centile is & 92.5263 & 92.8421 \\
\hline \% of cases below 99.6 centile is & 97.1579 & 96.9474 \\
\hline
\end{tabular}

Centile otherwise known as percentile has been one of the values of a statistical variable that divides the distribution of the variable into 100 groups having equal frequencies. The 99.6 percent of the values in the function $\alpha+\beta_{1} \cos \left(\mathrm{HR}_{2}\right)+\beta_{2} \sin \left(\mathrm{HR}_{1}\right)_{\text {lies at }} 97.1579$ centile, capturing and explaining the wave nature of the covariates above the 99.6 percent of the values in the function $\alpha+\beta_{1} \sin \left(\mathrm{HR}_{2}\right)+\beta_{2} \cos \left(\mathrm{HR}_{1}\right)$ that lies at 96.9474 centile in capturing and explaining the wave nature of the system.

\begin{tabular}{|c|c|c|c|}
\hline \multicolumn{2}{|c|}{$\alpha+\beta_{1} \cos \left(\mathrm{HR}_{2}\right)+\beta_{2} \sin \left(\mathrm{HR}_{1}\right)$} & \multicolumn{2}{|c|}{$\alpha+\beta_{1} \sin \left(\mathrm{HR}_{2}\right)+\beta_{2} \cos \left(\mathrm{HR}_{1}\right)$} \\
\hline Parameter & Estimate & Parameter & Estimate \\
\hline Mean & 0.0057 & Mean & -0.0056 \\
\hline Variance & 1.0010 & Variance & 1.0011 \\
\hline coef. of skewness & 1.5899 & coef. of skewness & 1.5633 \\
\hline $\mathrm{FCC}$ & 0.9437 & $\mathrm{FCC}$ & 0.9449 \\
\hline Cox-Snell & 0.85 & Cox-Snell & 0.54 \\
\hline Cragg-Uhler & 0.85 & Cragg-Uhler & 0.54 \\
\hline
\end{tabular}

Table. 4 divulged the approximately equivalence of the residual variance of the two alternate wave nature of Cosine and Sine equations as well as the same residual location parameter of positive effect by $\alpha+\beta_{1} \cos \left(\mathrm{HR}_{2}\right)+\beta_{2} \sin \left(\mathrm{HR}_{1}\right)$, whereas $\alpha+\beta_{1} \sin \left(\mathrm{HR}_{2}\right)+\beta_{2} \cos \left(\mathrm{HR}_{1}\right)$ adopted the negative effect of location parameter. In addition, the two alternate equations were not affected by skewedness (outliers), since their skewedness coefficients of 1.5899 and 1.5633 respectively are < 3. The Filliben Correlation Coefficient (FCC), which is use as test statistic for normal probability correlation coefficient of composite hypothesized for normality (non-normal) test; since its coefficient $r \approx 0.940$ for the two equations, it implies the noisy data indicated a length of lower tail (symmetric shorter-tailed) of $94 \%$ with $5 \%$ level of significant as maintained by Filliben [19]. The CoxSnell residual and Cragg-Uhler coefficients of (0.85 and 0.54) and (0.85 and 0.54) respectively for assessing the goodness-of-fit for the heartbeats' regression hinted the formal fitted function accommodated the wave nature of the heartbeat of the babies to $85 \%$ and the latter was able to explain to $54 \%$. The two residual indexes are alternate index for the Pseudo- $R^{2}$.

\section{Conclusion}

From the anteceding, it is necessary to do ascertain the wavy trend of regressors (covariates) to throe the level of noisy and frequency tone. By doing so, it allows a clear-cut whether the distributional property of the noise is a trigonometry (Cosine, Sine) of two or more covariates linear regression. The rate of heartbeats exactly after an hour, exactly after two hours and exactly three hours after birth followed a noisy Cosine and Sine wave nature trigonometry regression. The alternate Cosine and Sine two covariates was subjected to the heartbeats' observations such that the fitted equation of $\alpha+\beta_{1} \cos \left(\mathrm{HR}_{2}\right)+\beta_{2} \sin \left(\mathrm{HR}_{1}\right)$ captured the wave nature than the alternate function of $\alpha+\beta_{1} \sin \left(\mathrm{HR}_{2}\right)+\beta_{2} \cos \left(\mathrm{HR}_{1}\right)$. It is to be noted that this research could be extended to more than two co-variates linear trigonometry regression, that is generalized multiple trigonometry regression.

\section{References}

[1] Dette, H., Neumeyer, N., and Pilz, K. F., 2006. "A simple non-parametric estimator of a monotone regression function." Bernoulli, vol. 12, pp. 469- 490.

[2] Prahutama, A., Suparti, D., and Utami, T. W., 2018. "Modeling fourier regression for time series data- a case study: Modeling inflation in foods sector in indonesia. Iop conference series." Journal of Physics, vol. 974, p. 012067. 
[3] Shyti, B., Isa, I., and Paralloi, S., 2016. "Multiple regressions for the financial analysis of alabanian economy." Academic Journal of Interdisciplinary Studies MCSER Publishing, vol. 5, pp. 2281- 4612.

[4] Brown, L. D. and Levine, M., 2007. "Variance estimation in nonparametric regression via the difference sequence method." The Annals of Statistics, vol. 35, pp. 2219 -2232.

[5] Brunel, N. J.-B., 2008. "Parameter estimation of ODE's via non-parametric Estimators." Electronic Journal of Statistics, vol. 2, pp. 1242-1267.

[6] German, S. and Hwang, C.-R., 1982. "Non-parametric maximum likelihood estimation by method of sieves." The Annals of Statistics, vol. 10, pp. 401-414. Available: htpp://www.jstor.org/stable/224065

[7] Moliere, N.-M. and Alexandre, B., 2015. "Semi-parametric maximum likelihood method for interaction in case-mother control-mother designs: Package spmlficmcm." Journal of Statistical Software, vol. 68, pp. 117.

[8] Drapper, N. R. and Smith, H. D., 1992. Applied regression analysis. 2nd ed. New York: Marcel Dekker.

[9] Valveny, N. and Gilliver, S., 2016. "How to interpret and report the results from multivariable analyses." Medical Writing, vol. 25, pp. 37-42. Available: www.emwa.org

[10] Hanley, J. A., 2016. "Simple and multiple linear regression: Sample size considerations." Journal of Clinical Epidemiology, vol. 79, pp. 112-119.

[11] Eubank, R. L., 1990. "Trigonometric series regression estimators with an application to partially linear models." Journal of Multivariate Analysis, vol. 32, pp. 70-83.

[12] Neath, A. and Cavanaugh, J., 1997. "Regression and time series model selection using variants of the schwarz information criterion." Communications in Statistics Theory and Methods, vol. 26, pp. 559-580.

[13] Ostertagová, E. and Ostertag, O., 2015. "Regression analysis and seasonal adjustment of time series." Journal of Automation and Control, vol. 3, pp. 118-121.

[14] Wilcox, R., 2017. "The regression smoother LOWESS: A confidence band that allows heteroscedasticity and has some specified simultaneous probability coverage." Journal of Modern Applied Statistical Methods, vol. 16 , pp. 29-38.

[15] Giurcaneanu, C. D., 2007. Estimation of sinusoidal regression models by stochastic complexity. ICASSP 2007, Honolulu, Hawaii, USA.

[16] Montgomery, D. C., 2013. Introduction to statistical quality control. 7th ed. New York, NY, USA: John Wiley and Sons.

[17] Yu, Y. and Ruppert, D., 2002. "Penalized spline estimation for partially linear single-index models." Journal of the American Statistical Association, vol. 97, pp. 1042-1054.

[18] Rigdon, E., Turabelidze, G., and Jahanpour, E., 2014. "Trigonometric regression for analysis of public health surveillance data." Journal of Applied Mathematics, vol. 3, pp. 673-293. Available: http://dx.doi.org/10.1155/2014/673293

[19] Filliben, J. J., 1975. "The probability plot correlation coefficient test for normality." Technometrics, vol. 17, pp. 111-117. Available: htpp://www.jstor.org/stable/1268008 